Casos Clínicos

Arch. Esp. Urol., 60, 9 (1.137-1.140), 2007

\section{METÁSTASIS EN SENOS PARANASALES SECUNDARIA A ADENOCARCINOMA PROSTÁTICO.}

Roberto Llarena Ibarguren, Jorge García-Olaverri Rodríguez, Ainara Villafruela Mateos, Igor Azurmendi Arin, Ivan Olano Grasa y Carlos Pertusa Peña.

Servicio de Urología. Hospital de Cruces. Baracaldo. Vizcaya. España.

Resumen.- OBJETIVO: Presentar un nuevo caso de metástasis excepcional por carcinoma de próstata.

MÉTODO: Varón de 64 años diagnosticado 9 meses antes de carcinoma prostático diseminado, en tratamiento hormonal además de bifosfonatos, que debuta con elevación de las cifras de PSA, disestesia facial y exoftalmos izquierdo. En RMN se reconoce la existencia de una masa sólida en seno maxilar derecho con afectación del suelo orbitario del mismo lado, y de otra de las mismas características localizada en seno frontal izquierdo con invasión del techo de la órbita homolateral y afectación extraconal orbitaria.
RESULTADOS: Una vez establecido el diagnóstico se sometió al paciente a maniobras hormonales y se administró quimioterapia con docetaxel obteniendo una estabilización de la enfermedad mensurable con una remisión bioquímica de los niveles del PSA al principio del tratamiento, para progresar posteriormente, sin observarse cambios en las imágenes metastásicas. En la actualidad se ha iniciado de nuevo terapia citorreductora con docetaxel.

CONCLUSIONES: El 1\% de los tumores prostáticos afectarán la cabeza en su evolución. Las metástasis más frecuentes se localizan en cerebro y meninges, siendo la afectación de senos paranasales y órbita ocular extraordinaria. La importancia de éstas viene dada por la afectación en vecindad de la órbita y del ojo. Serán precisas para su control las maniobras hormonales de $2^{a}$ línea, la radioterapia local y la quimioterapia sistémica, aunque con resultados desalentadores. En general el pronóstico es malo, con sobrevivas cortas.

Palabras clave: Próstata. Metástasis. Seno paranasal.

Summary.- OBJECTIVE: To report a new case of exceptional metastases from a prostatic carcinoma.

METHODS: 64-year-old male with nine months history of disseminated prostate cancer, taking hormonal treatment and biphosphonates, who presents with rising PSA, facial dysesthesia and left exophtalmos. MRI recognizes the existence of a solid mass in the right maxillary sinus with involvement of the ipsilateral orbital floor, and another one in the left frontal sinus invading the roof of the ipsilateral orbit and also with orbital extraconal involvement.

RESULTS: Once the diagnosis was established hormonal maneuvers were performed and chemotherapy with docetaxel was administered achieving at the start of treatment measurable disease stabilization with biochemical remission of PSA levels, followed posteriorly by progression without changes in the metastatic images. Currently new cytoreductive therapy with docetaxel has been initiated.

CONCLUSIONS: $1 \%$ of the prostatic tumors involve the head in their evolution. Most frequent metastases are localized in the brain and meninges, being the involvement of paranasal sinuses and ocular orbit extraordinary. The importance of these comes from the extension to the orbit and the eye in vicinity. Second line hormonal maneuvers, local radiotherapy and systemic chemotherapy will be necessary for control, although results are discouraging. In general, prognosis is poor, with short survival.

Keywords: Prostate. Metastases. Paranasal sinus.
Roberto Llarena Ibarguren

Apartado de Correos 20134

48080 Bilbao. (España)

rllarena@eusklanet.net 


\section{INTRODUCCIÓN}

En la actualidad tan sólo entre el 20 y el $30 \%$ de los tumores prostáticos se encuentran diseminados en el momento del diagnóstico. La diseminación clásica es a ganglio linfático, hueso y pulmón. Aunque en la mayoría la evolución es previsible, algunos casos presentarán un curso evolutivo variable e impredecible (1). Así, el $1 \%$ de los tumores malignos de la próstata afectan cabeza y cuello durante su diseminación (2), siendo la localización en las leptomeninges la más frecuente. Existen autores que refieren tan sólo un $0^{\prime} 2 \%$ de diagnósticos en pacientes vivos (3), siendo el $1 \%$ cifras resultantes de estudios autópsicos.

En general, la metástasis intracraneal va precedida del diagnóstico previo tumoral (4), aunque hay casos excepcionales en los que ésta es el debut del proceso maligno (2).

\section{CASO CLÍNICO}

Varón de 64 años que consultó en mayo de 2006 por disestesia facial y ptosis palpebral izquierda y proptosis del globo ocular del mismo lado.

Antecedentes personales de hipertensión arterial en tratamiento médico, intervenido de ulcus duodenal 20 años antes, y de poliposis laríngea en dos ocasiones en 1993 y 1994.

Diagnosticado de adenocarcinoma prostático mediante biopsia ecodirigida en septiembre de 2005, con PSA de 794, fosfatasas alcalinas de 870, y con rastreo óseo que evidenció múltiples metástasis óseas. En esa época se instauró tratamiento mediante análogos de Ih-rh, flutamida y ácido zoledrómico $4 \mathrm{mg}$ cada 21 días durante

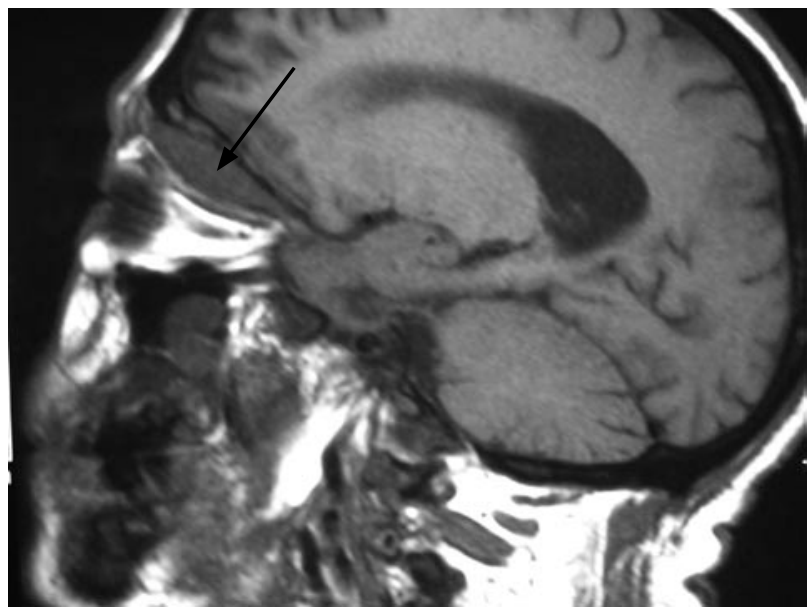

FIGURA 1. RMN cerebral. Corte sagital. Ocupación de seno frontal izdo. Masa sólida que afecta la órbita.
6 meses. El nadir de PSA se alcanzó en enero de 2006 con cifras de $36^{\prime} 17 \mathrm{ng} / \mathrm{ml}$.

Días antes del ingreso refería adormecimiento facial derecho desde el pómulo hasta el labio superior, y simultáneamente ptosis palpebral izquierda junto a protrusión ocular. No aquejaba diplopía, dolor facial ni ocular.

En la exploración física el paciente estaba consciente, orientado y colaborador, presentando ptosis palpebral izquierda con salida del ojo del mismo lado, sin soplo aparente, con paresia del III y VI pares izquierdos. Asimismo presentaba alteraciones en la sensibilidad de la mitad derecha de la cara, siendo el resto de la exploración neurológica motora y sensitiva normal.

Dentro de las pruebas complementarias, en sangre destacaba GGT de 86, fosfatasa alcalina de 1145, y un PSA de $121^{\prime} 02$.

Se realizó RMN cerebral y angio RMN que demostraron lesiones isquémicas supratentoriales y masa orbitaria de localización extraconal pegada a techo orbitario izquierdo que desplaza y engloba caudalmente el músculo recto superior, a la vez que existe una ocupación del seno frontal izquierdo por una masa de iguales características a la descrita. El reborde orbitario superior permanece íntegro sin aparentes datos de infiltración. La obliteración de la grasa de la grasa extraconal superior condiciona desplazamiento inferior y proptosis ocular (Figura 1). Asimismo existe una masa expansiva localizada en seno maxilar derecho que rompe la pared lateral extendiéndose hacia partes blandas, provocando interrupción del suelo de la órbita derecha e invasión del espacio extraconal inferior, desplazando sin invadirlo, el músculo recto inferior (Figura 2). Los senos cavernosos se encontraban libres.

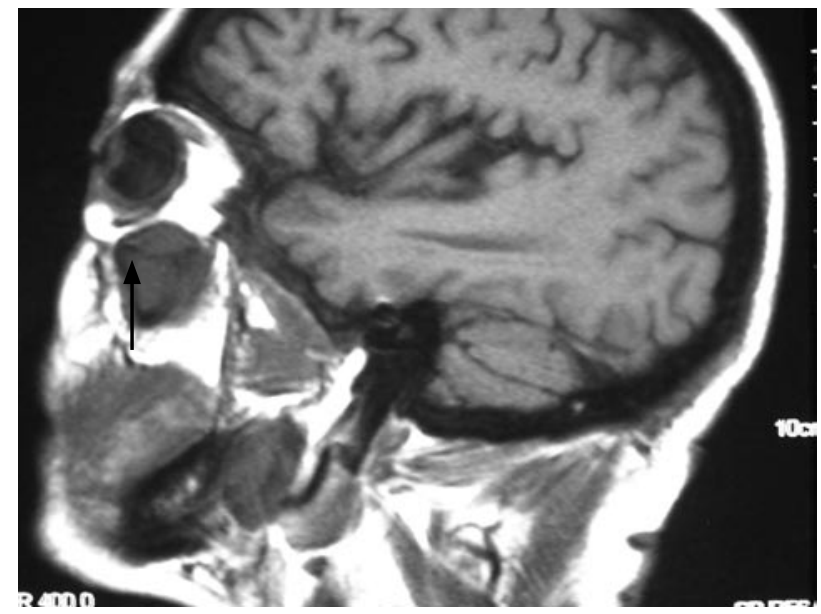

FIGURA 2. RMN cerebral. Corte sagital. Masa sólida que ocupa el seno maxilar derecho y que rompe el suelo de la órbita ipsilateral afectando espacio extraconal inferior. 


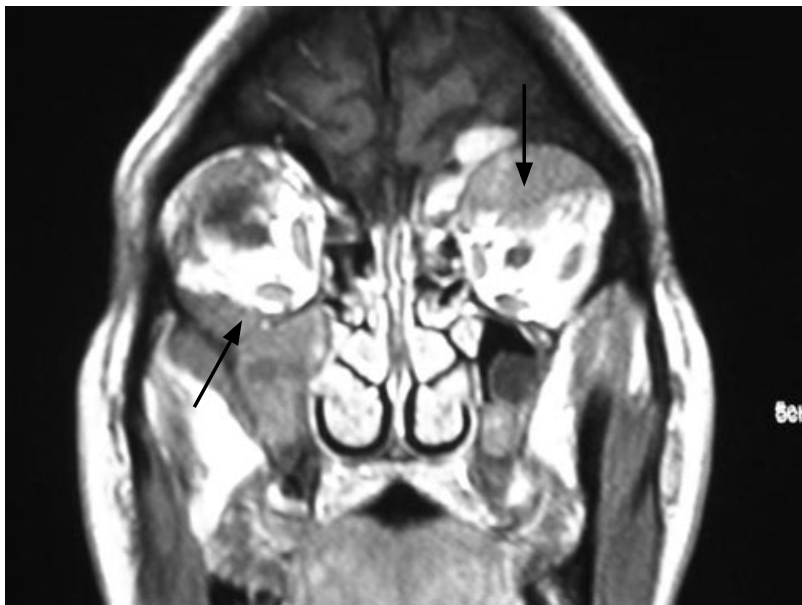

FIGURA 3. RMN cerebral. Corte coronal. Se aprecian en la misma imagen las afectaciones maxilar derecha y frontal izquierda.

En la tomografía axial computerizada toraco-abdominopélvica se diagnosticó de patrón óseo con infiltración blástico-lítica vertebral, sin alteraciones viscerales.

Se diagnosticó de metástasis de adenocarcinoma prostático en seno maxilar derecho y seno frontal izquierdo, con afectación orbitaria extraconal inferior derecha y superior izquierda.

Se desechó la radioterapia local por el riesgo de ceguera. Como tratamiento se retiró la flutamida, manteniéndose el análogo de lh-rh. En junio de 2006 se inicia tratamiento con docetaxel $70 \mathrm{mg} / \mathrm{m} 2$ cada 21 días, junto con prednisona $10 \mathrm{mg}$ día. Se completaron 6 ciclos hasta finales de septiembre de 2006.

Se alcanza un nadir de PSA en julio de 2006 con cifras de $6^{\prime} 8 \mathrm{ng} / \mathrm{ml}$, tras las dos primeras administraciones de docetaxel. El paciente refiere disminución de la proptosis ocular izquierda. Se completan 6 ciclos, con excelente tolerancia. En octubre de 2006 el PSA es de 149 $\mathrm{ng} / \mathrm{ml}$, no habiendo variado las imágenes en $\mathrm{RMN}$. Recientemente en enero de 2007 se reinicia de nuevo tratamiento con docetaxel y prednisona.

\section{COMENTARIOS}

El $1 \%$ de los tumores malignos presentan metástasis en área maxilofacial (5). Las más frecuentes afectarán al ojo y tejidos vecinos. Las primeras descripciones de metástasis en órbita ocular se deben a Horner en 1864 y a Perl en 1872 (6). El origen tumoral más frecuente es la leucemia y los linfomas como los más representativos en frecuencia, seguidos de los carcinomas de mama y pulmón, del melanoma y de los tumores genitourinarios y gastrointestinales (7).
Topográficamente el asiento tumoral dentro de la órbita más frecuente es intraocular, ya sea en el propio ojo o en tejidos perioculares (6). La lesión orbitaria ósea con afectación secundaria es mucho más rara. En los casos publicados en nuestro país la mayoría de las metástasis retroorbitarias asientan en senos paranasales, etmoidales, frontales, esfenoidales o maxilares desde donde afectan estructuras oculares por destrucción del techo o del suelo orbitarios $(1,2,9,10)$. En este sentido hay citas que refieren que las metástasis retroorbitarias son producidas en segundo lugar por el carcinoma prostático (11). Sin embargo también hay autores (12) que opinan que en la mayoría de los casos la lesión ósea es precursora de la afectación de estructuras adyacentes, no constituyendo la órbita ocular una excepción.

En nuestro caso la localización primaria fueron los senos maxilar derecho y frontal izquierdo, desde donde se afectó la órbita y tejidos extraconales. Recientemente Torres (13) ha descrito un caso de metástasis en seno maxilar secundario a adenocarcinoma renal, que presentaba erosión ósea adyacente. En este sentido Jiménez en 2001 (10) describía que las metástasis en senos paranasales y fosas son excepcionales, evaluando en unos 50 los casos publicados. El mismo autor otorgaba al adenocarcinoma renal el primer puesto de los tumores que con mayor frecuencia afectaban la región nasosinusal, seguidos de los carcinomas de mama y pulmón.

La mayoría de los autores coinciden en que la vía de diseminación más lógica se realiza por el torrente circulatorio a través del plexo venoso paravertebral de Batson. Esta es una red anastomótica de venas carentes de válvulas que circundan la médula espinal, interconectadas con las grandes venas pelvianas, intercostales, ácigos y cava. De esta manera el filtro pulmonar queda evitado, pudiendo aparecer metástasis en cabeza y cuello en ausencia de diseminación pulmonar (8).

Clínicamente pueden cursar con diplopía por afectación de pares craneales,, exoftalmos, disminución de la agudeza visual, dolor, eritema e hinchazón ocular, síntomas todos ellos de rápida instauración. También pueden aparecer epistaxis, insuficiencia nasal respiratoria, algias faciales y frontales, rinorrea purulenta, y demás síntomas derivados de la afectación sinusal (8).

Para su diagnóstico los métodos actuales de imagen como la resonancia magnética nuclear, la angiorresonancia y la tomografía axial computerizada ofrecen diagnósticos precisos tanto de la localización y afectación de estructuras vecinas como de las características radiológicas de las lesiones.

Aunque las pruebas de imagen son lo suficientemente precisas, en ocasiones se podrá presentar el diagnóstico diferencial con lesiones tumorales del tipo condrosarcoma, meningioma óseo, osteoma, ostesarcoma, osteoblastoma, fibroma osificado incluso con el Paget 
monoostótico (8), o con lesiones vasculares como aneurismas carotídeos, fístulas carótido-cavernosas etc.

El diagnóstico de confirmación en cuanto al origen histológico lo realizará lógicamente la biopsia o la punción aspiración $(8,10)$. En el caso de que existieran dudas del origen se pueden realizar técnicas inmunohistoquímicas. En aquellos casos en los que se conozca la existencia del carcinoma primario no suele hacer falta la confirmación histológica (2).

En lo referente al tratamiento en los casos de diagnóstico primario se impone el bloqueo hormonal androgénico. Por otra parte cuando la progresión es evidente habrá que realizar maniobras hormonales de segunda línea, retirada o adicción de antiandrógeno, fosfato de estramustina, bicalutamida a dosis altas, e incluso administración de docetaxel junto a prednisona.

En aquellos casos de metástasis únicas se puede indicar la cirugía junto a la radioterapia y hormonoterapia, pudiendo alcanzarse en ciertos casos supervivencias de hasta 3 años (3). Caso de indicarse cirugía será precisa la confirmación histopatológica previa, pudiéndose realizar embolización prequirúrgica (13).

La radioterapia local a altas dosis puede lograr el control de la enfermedad hasta en el $90 \%$ de los casos cuando la lesión es de reciente aparición, en general menos de un mes (12). Cuando la lesión tiene más evolución, mayor de 3 meses, la radioterapia local tan solo logrará el control en el $25 \%$ de los casos (12).

El pronóstico salvo en casos de metástasis aislada, y cuando la lesión es la precursora del diagnóstico de carcinoma prostático, es infausto (1), a corto plazo, con supervivencias inferiores al año en la mayoría de las publicaciones, salvo en la de Olivier que reportó un caso con una sobrevida de 16'3 meses (8).

\section{BIBLIOGRAFÍA y LECTURAS RECOMENDADAS ( ${ }^{*}$ lectura de interés $y^{* *}$ lectura fundamental)}

*1. GILSANZ, M.; GONZALEZ, C.; RONCALES, A. y cols.: "Metástasis en seno maxilar como presentación de una adenocarcinoma prostático". Actas Urol. Esp., 16: 272, 1992.

*2. BONILLO, M.A.; QUEIPO, J.A.; PALMERO, J.A. y cols.: "Metástasis retroorbitaria como manifestación inicial del cáncer de próstata”. Actas Urol. Esp., 27: $562,2003$.

3. LYNES, W.L.; BOSTWICK, D.G.; FREIHA, F.S. y cols.: "Parenchymal brain metastases from adenocarcinoma of prostate". Urology, 28: 280, 1986.

4. SUTTON, M.A.; WATKINS, H.L.; GREEN, L.K. y cols.: "Intracranial metastases as first manifestation of prostate cancer". Urology, 48: 789, 1996.

5. BHASAR, S.N.: "Synopsis of oral pathology". St. Louis. CV Mosby: 374, 1973.

**6. BLOCH, R.S.; GARDNER, S.: "The incidence of ocular metastatic carcinoma". Arch. Opthal., 85: 673, 1971.

7. FERRY, P.A.; FONT, R.L.: "Carcinoma metastatic to the eye and orbit. A clinicopathological study of 227 cases". Arch. Ophtal., 92: 276, 1974.

**8. OLIVIER, C.; LAO, A.H.; SALAS, C. y cols.: "Metastasis de origen prostatico en tejidos blandos periorbitarios". Actas Urol. Esp., 16: 79, 1992.

9. PIERAS, E.; ROSALES, A.; LÓPEZ, H. y cols.: "Metástasis intracraneales en cáncer de próstata". Actas Urol. Esp., 24: 626, 2000.

**10. JIMÉNEZ OLIVER, V.; LAZARICH VALDES, A.; DÁVILA MORILLO, A. y cols.: "Metastasis frontoetmoidal de adenocarcinoma prostático. Presentación de un caso clínico y revisión de la literatura". Acta Otorrinolarigol. Esp., 52: 151, 2001.

11. ROMERO, P.; PELLUCH, A.; LOBATO, J.J. y cols.: "Exoftalmos por metastasis orbitaria de carcinoma prostático". Arch. Esp. Urol., 44: 191, 1991.

12. GREENBERG, H.S.; DECK, M.D.F.; VIKRAM, B. y cols.: "Metastases to the base of the skull: Clinical findings in 43 patients". Neurology, 31: 530, 1981.

13. TORRES MUROS, B.; SOLANO ROMERO, J.R.; RODRÍGUEZ BARÓ, J.G. y cols.: "Adenocarcinoma renal metastástico en seno maxilar". Actas Urol. Esp., 30: 954, 2006 